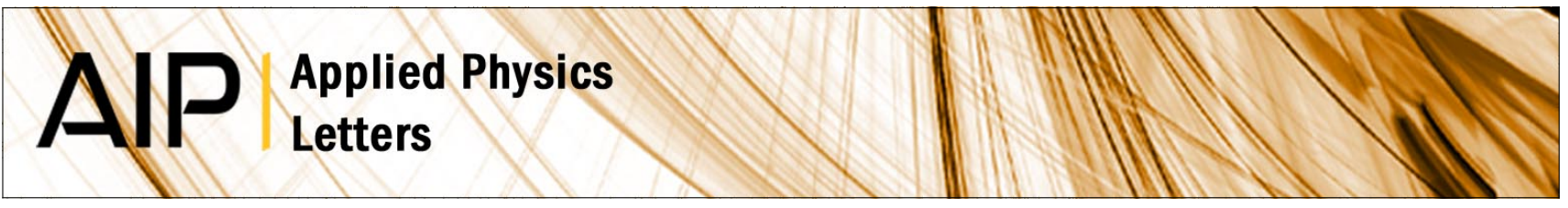

\title{
Gold substrate-induced single-mode lasing of GaN nanowires
}

Huiwen Xu, Jeremy B. Wright, Antonio Hurtado, Qiming Li, Ting-Shan Luk et al.

Citation: Appl. Phys. Lett. 101, 221114 (2012); doi: 10.1063/1.4768300

View online: http://dx.doi.org/10.1063/1.4768300

View Table of Contents: http://apl.aip.org/resource/1/APPLAB/v101/i22

Published by the American Institute of Physics.

\section{Related Articles}

High-power InP quantum dot based semiconductor disk laser exceeding 1.3W

Appl. Phys. Lett. 102, 092101 (2013)

Chirped InAs/InP quantum-dash laser with enhanced broad spectrum of stimulated emission

Appl. Phys. Lett. 102, 091102 (2013)

Semiconductor laser monolithically pumped with a light emitting diode operating in the thermoelectrophotonic regime

Appl. Phys. Lett. 102, 081116 (2013)

Determination of operating parameters for a GaAs-based polariton laser

Appl. Phys. Lett. 102, 081115 (2013)

GaN-based high contrast grating surface-emitting lasers

Appl. Phys. Lett. 102, 081111 (2013)

\section{Additional information on Appl. Phys. Lett.}

Journal Homepage: http://apl.aip.org/

Journal Information: http://apl.aip.org/about/about_the_journal

Top downloads: http://apl.aip.org/features/most_downloaded

Information for Authors: http://apl.aip.org/authors

\section{ADVERTISEMENT}

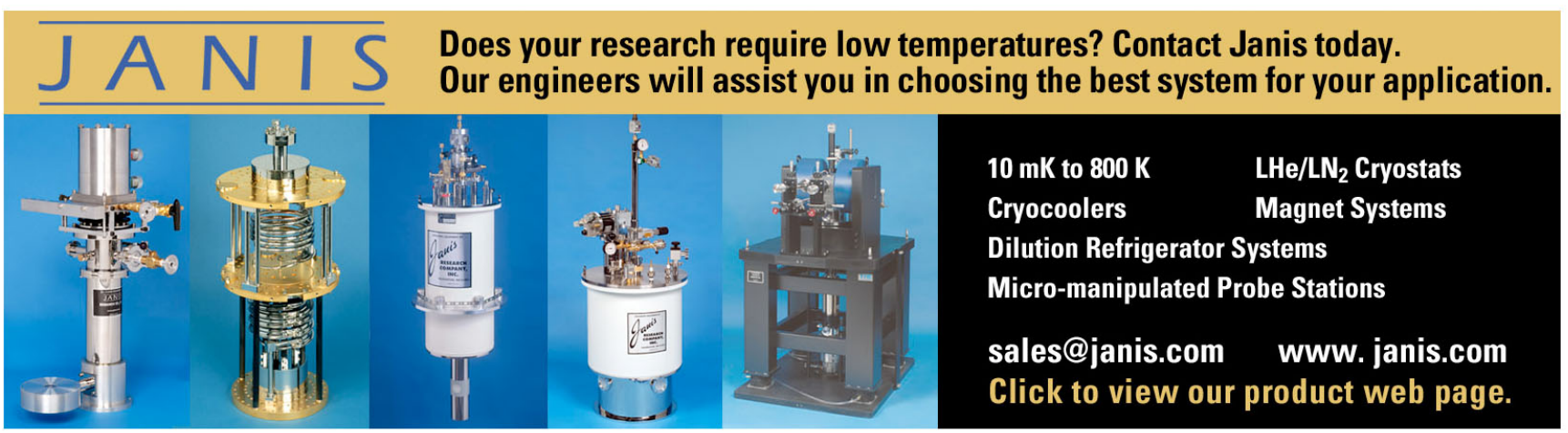




\title{
Gold substrate-induced single-mode lasing of GaN nanowires
}

\author{
Huiwen Xu, ${ }^{1}$ Jeremy B. Wright, ${ }^{1,2}$ Antonio Hurtado, ${ }^{1,3}$ Qiming Li, ${ }^{2}$ Ting-Shan Luk, ${ }^{2,4}$ \\ Jeffrey J. Figiel, ${ }^{2}$ Karen Cross, ${ }^{2}$ Ganesh Balakrishnan, ${ }^{1}$ Luke F. Lester, ${ }^{1}$ Igal Brener, ${ }^{2,4}$ \\ and George T. Wang ${ }^{2, a)}$ \\ ${ }^{1}$ Center for High Technology Materials, University of New Mexico, Albuquerque, New Mexico 87106, USA \\ ${ }^{2}$ Sandia National Laboratories, Albuquerque, New Mexico 87185, USA \\ ${ }^{3}$ School of Computer Science and Electronic Engineering, University of Essex, Wivenhoe Park, \\ Colchester CO4 3SQ, United Kingdom \\ ${ }^{4}$ Center for Integrated Nanotechnology, Sandia National Laboratories, Albuquerque, New Mexico 87185, USA
}

(Received 27 September 2012; accepted 6 November 2012; published online 27 November 2012)

We demonstrate a method for mode-selection by coupling a GaN nanowire laser to an underlying gold substrate. Multimode lasing of GaN nanowires is converted to single-mode behavior following placement onto a gold film. A mode-dependent loss is generated by the absorbing substrate to suppress multiple transverse-mode operation with a concomitant increase in lasing threshold of only $\sim 13 \%$. This method provides greater flexibility in realizing practical single-mode nanowire lasers and offers insight into the design of metal-contacted nanoscale optoelectronics. (c) 2012 American Institute of Physics. [http://dx.doi.org/10.1063/1.4768300]

Semiconductor nanowire lasers have attracted intense interest as promising coherent light sources for on-chip applications. ${ }^{1-4}$ Many of these applications, such as imaging, multiplex communication, and data storage, require high beam quality, and spectral purity. These conditions can be satisfied if single-mode operation is achievable in nanowire lasers. However, due to the lack of mode selection mechanisms, most reported nanowire lasers exhibit multimode behavior. ${ }^{5,6} \mathrm{~A}$ way of addressing this issue is to make use of coupled cavities, ${ }^{7-9}$ which can generate a mode selection mechanism by the Vernier effect. However, this approach requires complicated nano-manipulation to form the coupled cavities. Single-mode, as-fabricated Fabry-Perot GaN nanowire lasers have also been successfully demonstrated by precise control of the nanowire geometry (diameter and length) to reduce the number of transverse and longitudinal modes. ${ }^{10}$ However, this approach limits the GaN nanowire diameter to $<\sim 130 \mathrm{~nm}$ and length to $<\sim 6 \mu \mathrm{m}$, which could limit nanolaser design flexibility and reduce cavity gain, and could also potentially increase the surface-related defects and surface depletion effects from the higher surface-to-volume ratio. ${ }^{11}$

In this letter, we demonstrate an alternative technique to realize single-mode nanowire lasers by coupling GaN nanowire to a metal substrate. Transverse mode selection is achieved through the contact of the nanowires with a gold substrate, which generates a mode-sensitive ohmic loss and thus enables single transverse-mode operation. Onedimensional length control of the nanowires is utilized for multiple longitudinal-mode suppression. ${ }^{10}$ Using this technique, single-mode emission at $\sim 369 \mathrm{~nm}$ of a $\sim 350 \mathrm{~nm}$ diameter nanowire is demonstrated with a side-mode suppression rate (SMSR) of $17.4 \mathrm{~dB}$ and a bandwidth of $0.12 \mathrm{~nm}$ (the resolution limit of the spectrometer). Our results provide guidance for the design of nanolasers and offer a practical method for realizing single-mode nanoscale light-emitting devices.

\footnotetext{
${ }^{\text {a) }}$ Author to whom correspondence should be addressed. Electronic mail: gtwang@sandia.gov.
}

GaN nanowire lasers typically exhibit multiple transverse-mode and multiple longitudinal-mode oscillation. ${ }^{9}$ In order to obtain single-mode operation, it is necessary to introduce a multimode suppression technique to the nanowire cavity. Previously, we have demonstrated that multiple longitudinal-mode oscillation in GaN nanowires can be suppressed by reducing the nanowire length to $\sim 5 \mu \mathrm{m}$, at which point the mode competition is strongly enhanced in the cavity. ${ }^{10}$ Here, we concentrate our efforts on the issue of multiple transverse-mode suppression. Single transverse-mode GaN nanowire lasers have been demonstrated, but this approach requires diameters below a critical diameter of $\sim 130 \mathrm{~nm}^{10}$ Transverse-mode selection techniques in macroscopic lasers, such as placing an intra-cavity pinhole into a bulk laser cavity, ${ }^{12,13}$ have been widely studied. In principle, this technique could work for nanowire lasers but would be difficult to achieve due to the small cavity volume and potential material damage from lithographic or electron and ion-beam nanostructuring methods. Alternatively, the light field inside a nanowire waveguide can be manipulated by affecting the evanescent wave of the waveguide modes, so as to generate an attenuation effect. ${ }^{14,15}$ Here, we propose a transversemode selection technique by means of an absorptive material serving as the nanowire substrate. Due to moderate ohmic loss in the ultraviolet band, gold was selected as a substrate material for achieving a single-mode GaN nanolaser. Note that this proposed on-metal dielectric nanolaser differs from the recently reported plasmonic nanolasers that rely on metallic guided modes. In the case of the plasmonic laser, the electric field is highly confined by the metallic structure. ${ }^{16-18}$ This contrasts from our proposed nanolaser since the mode is guided in the dielectric and the metal acts purely as a loss mechanism for high-order modes. Moreover, due to the difference in the spatial distributions, the cavity loss varies with different transverse modes, thereby generating the modedependent loss.

To investigate the proposed mode-dependent loss mechanism, we first conducted simulations to illustrate the modal 
properties of GaN nanowires placed on a gold substrate. The nanowire passive cavity eigenmodes were determined using a fully vectorial commercial mode solver from Lumerical, Inc., which has been widely used to analyze mode distribution, frequency response, and propagation loss of nanoscale waveguides. ${ }^{19}$ In this simulation, a cylindrical nanowire was defined on top of a gold substrate ( $200 \mathrm{~nm}$ thick). The operation wavelength was set as $370 \mathrm{~nm}$ ( $\mathrm{GaN}$ bandedge emission wavelength). The refractive index of $\mathrm{GaN}$ was assumed to be $2.6,^{20}$ and the real part and imaginary part of gold were set as 1.70 and 1.88 , respectively (these values correspond to a wavelength of $\sim 370 \mathrm{~nm}){ }^{21}$ The transverse modes supported by this geometry were analyzed by a 2-dimensional cross section calculation in a $1 \mu \mathrm{m} \times 1 \mu \mathrm{m}$ window (perfectly matched layer boundary conditions were used), where 200 unit cells in both $\mathrm{x}$ and $\mathrm{y}$ directions were employed to ensure the simulation accuracy. The dimensions of the nanowires were modified to study the substrate effect on nanowires with different geometries.

Fig. 1 shows the transverse mode distributions supported by a $300 \mathrm{~nm}$ diameter nanowire placed on the gold substrate. It is seen that ten modes are supported by this geometry. Due to the presence of the metal substrate, the mode degeneracy is broken, with modes in each order showing different polarization, mode distribution, and propagation loss (no propagation loss obtained from similar simulations of nanowires with a dielectric substrate). Table I shows the propagation loss for the different modes, showing that the propagation loss is sensitive to mode distribution, i.e., more absorption occurs for modes that have more of their modal fraction confined within the metal region. Mode 2 has the smallest propagation loss of $1730 \mathrm{~dB} / \mathrm{cm}$ (corresponding to an extra $1.83 \mathrm{~dB}$ or $34.4 \%$ cavity loss for a $5.3 \mu \mathrm{m}$ nanowire cavity), which is significantly lower than that of the other modes. Mode 3 has the second smallest loss of $4806 \mathrm{~dB} / \mathrm{cm}$, corresponding to an extra $5.09 \mathrm{~dB}$ or $84.7 \%$ cavity loss for a $5.3 \mu \mathrm{m}$ nanowire cavity. Therefore, single transverse-mode lasing is expected since mode 2 will be the only mode with a cavity loss that can be overcome by the modal gain. Further simulations were also conducted for nanowires with different diameters, and it was found that higher/lower losses will be generated with smaller/larger nanowire diameters. Regardless, in all cases a modedependent loss was always obtained. We have also found that nanowires with diameters ranging from $\sim 250$ to $\sim 400 \mathrm{~nm}$ are suitable for generating single transverse-mode
TABLE I. Propagation loss for the different modes supported by a $300 \mathrm{~nm}$ diameter nanowire (operation wavelength: $370 \mathrm{~nm}$ ).

\begin{tabular}{lccccc}
\hline \hline & Mode 1 & Mode 2 & Mode 3 & Mode 4 & Mode 5 \\
Loss $(\mathrm{dB} / \mathrm{cm})$ & 8151 & 1730 & 4806 & 7109 & 16041 \\
& Mode 6 & Mode 7 & Mode 8 & Mode 9 & Mode 10 \\
Loss $(\mathrm{dB} / \mathrm{cm})$ & 34858 & 7551 & 22205 & 29175 & 28706 \\
\hline \hline
\end{tabular}

operation since one specific mode experiences much less attenuation than the others.

$\mathrm{GaN}$ nanowires with the expected proper geometry for single-mode lasing operation were then fabricated and optically characterized to experimentally verify the modeling results. The GaN nanowires were fabricated by a top-down, two-step etch technique, detailed in Ref. 10. This technique is able to produce vertically aligned c-axis oriented nanowires with precisely controlled geometries from c-plane $\mathrm{GaN}$ epilayers, and the fabricated nanowires have straight and smooth sidewalls. Nanowires were intentionally fabricated with $\sim 300 \mathrm{~nm}$ diameter and $\sim 5.3 \mu \mathrm{m}$ length for the purpose of verifying the simulation results and bypassing the multiple longitudinal-mode oscillation, ${ }^{10}$ respectively. These nanowires were then transferred to $\mathrm{Si}_{3} \mathrm{~N}_{4}$ (300 nm thick) on silicon substrate photolithographically patterned with gold regions (200 nm thick and $120 \mu \mathrm{m}$ diameter) for optical characterization. The structure of the substrate is sketched in Fig. 2. The optical properties of the nanowires were characterized using a micro-photoluminescence setup. The pump laser used was a frequency-quadrupled Nd:YAG laser operating at $266 \mathrm{~nm}$ with a pulse duration of $400 \mathrm{ps}$ and a repetition rate of $10 \mathrm{kHz}$. The nanowire was excited using the pulsed laser through a $50 \times$ ultraviolet objective with a $5 \mu \mathrm{m}$ spot size, and the emission from the nanowire was collected by the same objective and analyzed using a CCD detector and a $300 \mathrm{~mm}$ spectrometer with a 1200 groove $/ \mathrm{mm}$ holographic grating.

As a baseline, the lasing properties of $\mathrm{GaN}$ nanowires on areas of the $\mathrm{Si}_{3} \mathrm{~N}_{4}$ film (without gold) were first investigated. Then, in order to examine the impact of the gold substrate on the lasing properties, these same nanowires were subsequently transferred onto a gold-coated area of the membrane with a piezo-electric driven tungsten probe. Fig. 3(a) shows a scanning electron microscope (SEM) image of a GaN nanowire $\sim 350 \mathrm{~nm}$ in diameter and $5.3 \mu \mathrm{m}$ in length lying on the $\mathrm{Si}_{3} \mathrm{~N}_{4}$ film. Below threshold, the nanowire showed a broadband
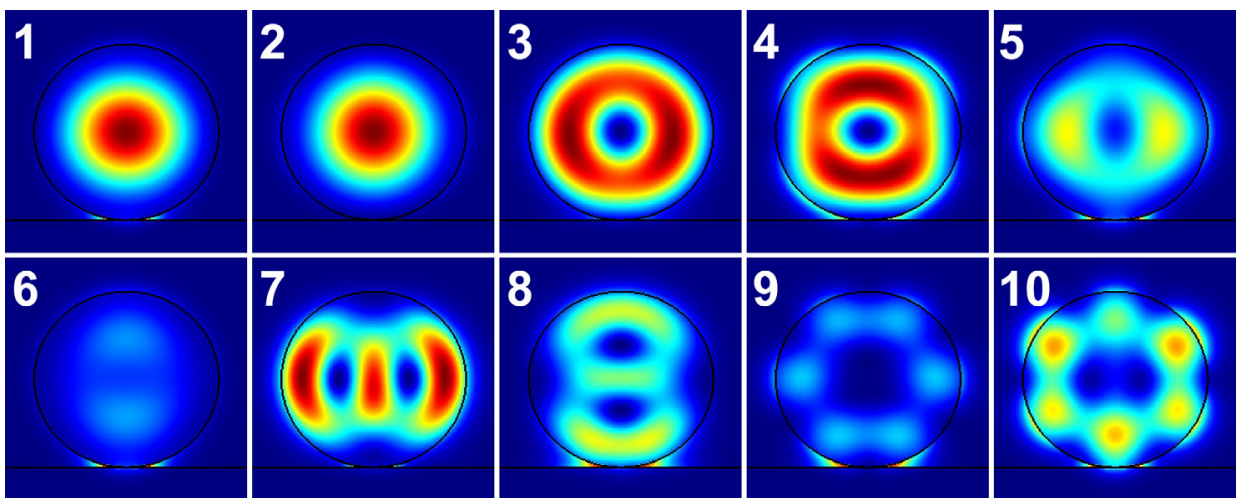

FIG. 1. Transverse modes supported by the nanowire-metal geometry. The black circle and straight line indicate the surfaces of the nanowire and metal substrate, respectively. 


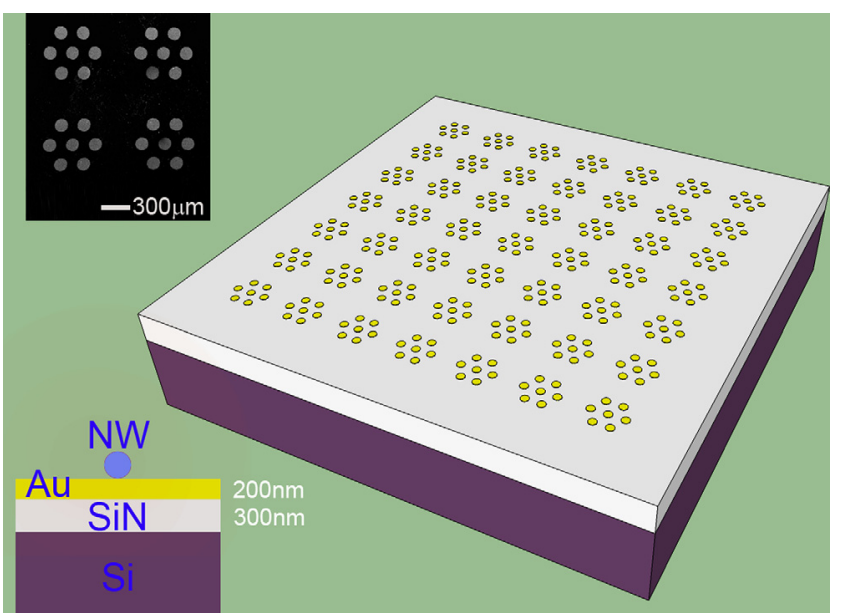

FIG. 2. Schematic showing the $\mathrm{Si}_{3} \mathrm{~N}_{4}$ film selectively patterned with gold spots for optical characterization of the $\mathrm{GaN}$ nanowires. The inset shows a SEM image of gold spots.

photoluminescence (PL) emission, with a spectrum centered at $367.5 \mathrm{~nm}$ and a full-width half-maximum of $\sim 7.5 \mathrm{~nm}$. Lasing was observed by gradually increasing the pump power with a threshold of $241 \mathrm{~kW} / \mathrm{cm}^{2}$. Fig. 4(a) shows typical lasing spectra at different pump powers. The blue curve shows the measured spectrum when the nanowire was pumped slightly below threshold, with several small peaks apparent. Upon increasing the pumping intensity beyond threshold, most of these peaks grew with an enhanced signal-to-noise ratio; moreover, new lasing peaks also emerged, as shown in the red spectral curve $\left(276 \mathrm{~kW} / \mathrm{cm}^{2}\right)$. The black curve shows the spectrum at a pump power of $458 \mathrm{~kW} / \mathrm{cm}^{2}$. Six primary peaks are seen in the spectrum with an uneven spectral spacing. A minimum spacing of $\sim 0.58 \mathrm{~nm}$ is observed, much smaller than the calculated longitudinal mode spacing of $\sim 2.1 \mathrm{~nm}$ for single transverse-mode operation. ${ }^{9}$ Taken together, these spectral features are indicative of multiple transverse-mode operation of the GaN nanowire laser. ${ }^{9}$

Fig. 3(b) shows the SEM image of the same nanowire after its transfer onto a gold-coated region of the substrate. Under gradually increased optical pumping, a similar spectral evolution from PL to lasing was observed, as seen in Fig. 4(b). Due to the extra cavity loss introduced by the metal substrate, the observed lasing threshold increased to $276 \mathrm{~kW} / \mathrm{cm}^{2}, \sim 13 \%$ larger than that observed directly on the
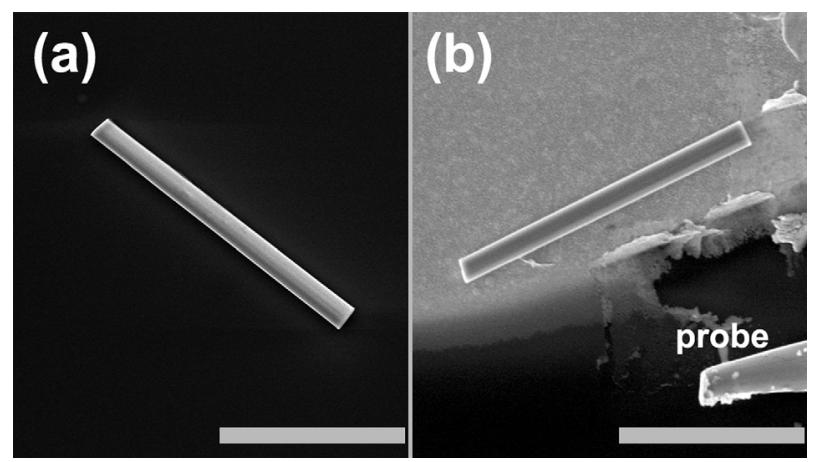

FIG. 3. SEM images of the same GaN nanowire (a) on top of the $\mathrm{Si}_{3} \mathrm{~N}_{4}$ film; and (b) after being transferred onto a gold-coated region. The scale bars represent $3 \mu \mathrm{m}$.
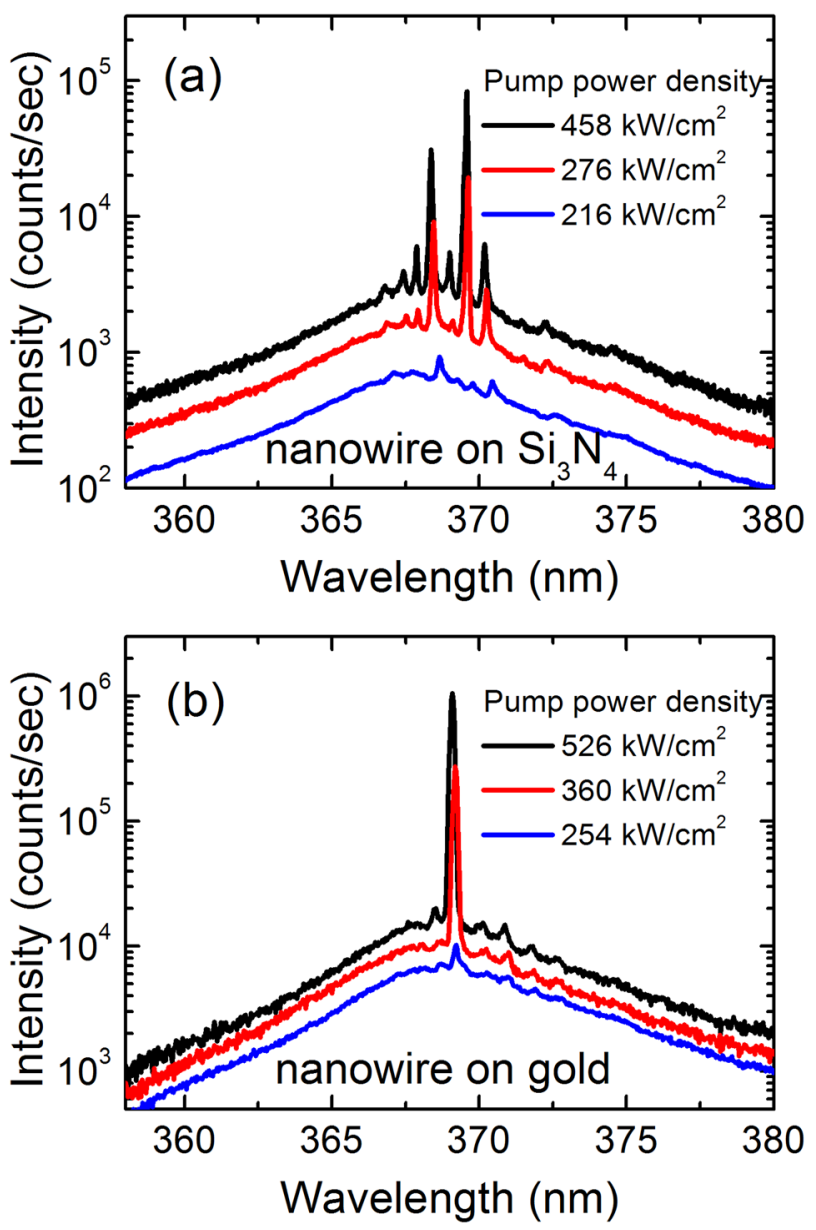

FIG. 4. Spectra of a GaN nanowire lasing (a) on $\mathrm{Si}_{3} \mathrm{~N}_{4}$ and (b) after being transferred onto gold.

$\mathrm{Si}_{3} \mathrm{~N}_{4}$ substrate. However, when pumped above the threshold, the nanowire placed on gold exhibited single-mode lasing behavior, distinctly different from the multimode lasing observed for the same nanowire on $\mathrm{Si}_{3} \mathrm{~N}_{4}$. The blue curve in Fig. 4(b) shows the emission of the nanowire when pumped slightly below threshold, exhibiting only one dominant peak at $369.3 \mathrm{~nm}$. This remains the case as the pump power increases above threshold (red and black curves). Under a pump intensity of $526 \mathrm{~kW} / \mathrm{cm}^{2}$, well above threshold, singlemode lasing with a SMSR of $17.4 \mathrm{~dB}$ and a bandwidth of $0.12 \mathrm{~nm}$, the resolution limit of the spectrometer, was measured (via Lorentzian fitting).

In order to further confirm this effect, the experiment was repeated with different nanowires whose diameters ranged from $\sim 250$ to $400 \mathrm{~nm}$. A similar transition from multimode to single-mode lasing operation was also observed when these nanowires were transferred from the $\mathrm{Si}_{3} \mathrm{~N}_{4}$ to the gold-covered regions. Thus, the predicted mode-sensitive loss mechanism induced by placement of the nanowire on the gold surface was confirmed.

In conclusion, we demonstrated a mechanism for lasingmode selection by coupling a GaN nanowire laser to an underlying gold substrate. A mode-dependent loss can be induced by the gold substrate to suppress multiple transverse-mode operation and thus enable single-mode lasing behavior from nanowires that otherwise exhibit multimode behavior, with an observed increase in lasing threshold of only $\sim 13 \%$. These 
results also offer guidance for the design of metal-contacted nanoscale devices and suggest that the impact of metal contacts on the optoelectronic properties may not be as detrimental as suspected. This method should provide more flexibility in realizing practical single-mode nanowire lasers compared to alternative methods requiring nanomanipulation of nanowires into coupled cavities or tight restrictions on the maximum nanowire diameter.

This work was supported by Sandia's Solid-StateLighting Science Energy Frontier Research Center, funded by the U.S. Department of Energy, Office of Science, Office of Basic Energy Sciences and Sandia's Laboratory Directed Research and Development program. Dr. Antonio Hurtado is funded by the European Commission under the Programme FP7 Marie Curie International Outgoing Fellowships (IOF) Grant No. PIOF-GA-2010-273822. This work was performed, in part, at the Center for Integrated Nanotechnologies, a U.S. Department of Energy, Office of Basic Energy Sciences user facility. Sandia National Laboratories is a multi-program laboratory managed and operated by Sandia Corporation, a wholly owned subsidiary of Lockheed Martin Corporation, for the U.S. Department of Energy's National Nuclear Security Administration under Contract No. DE-AC04-94AL85000.

${ }^{1}$ M. S. Gudiksen, L. J. Lauhon, J. Wang, D. C. Smith, and C. M. Lieber, Nature 415(6872), 617 (2002).

${ }^{2}$ Y. Li, F. Qian, J. Xiang, and C. M. Lieber, Mater. Today 9(10), 18 (2006).
${ }^{3}$ Y. Xia, P. Yang, Y. Sun, Y. Wu, B. Mayers, B. Gates, Y. Yin, F. Kim, and H. Yan, Adv. Mater. 15(5), 353 (2003).

${ }^{4}$ P. Yang, R. Yan, and M. Fardy, Nano Lett. 10(5), 1529 (2010).

${ }^{5}$ M. H. Huang, S. Mao, H. Feick, H. Yan, Y. Wu, H. Kind, E. Weber, R. Russo, and P. Yang, Science 292(5523), 1897 (2001).

${ }^{6}$ J. C. Johnson, H. J. Choi, K. P. Knutsen, R. D. Schaller, P. Yang, and R. J. Saykally, Nature Mater. 1(2), 106 (2002).

${ }^{7}$ Y. Xiao, C. Meng, P. Wang, Y. Ye, H. Yu, S. Wang, F. Gu, L. Dai, and L. Tong, Nano Lett. 11(3), 1122 (2011).

${ }^{8}$ Y. Xiao, C. Meng, X. Wu, and L. Tong, Appl. Phys. Lett. 99(2), 023109 (2011).

${ }^{9}$ H. Xu, J. B. Wright, T. S. Luk, J. J. Figiel, K. Cross, L. F. Lester, G. Balakrishnan, G. T. Wang, I. Brener, and Q. Li, Appl. Phys. Lett. 101(11), 113106 (2012).

${ }^{10}$ Q. Li, J. B. Wright, W. W. Chow, T. S. Luk, I. Brener, L. F. Lester, and G. T. Wang, Opt. Express 20(16), 17873 (2012).

${ }^{11}$ Q. Li and G. T. Wang, Nano Lett. 10(5), 1554 (2010).

${ }^{12}$ M. A. Hadley, G. C. Wilson, K. Y. Lau, and J. S. Smith, Appl. Phys. Lett. 63(12), 1607 (1993)

${ }^{13}$ E. W. Young, K. D. Choquette, S. L. Chuang, K. M. Geib, A. J. Fischer, and A. A. Allerman, IEEE Photonics Technol. Lett. 13(9), 927 (2001).

${ }^{14}$ K. Kieu and M. Mansuripur, Opt. Lett. 32(15), 2242 (2007).

${ }^{15}$ Y. Song, K. Morimune, S. Y. Set, and S. Yamashita, Appl. Phys. Lett. 90(2), 021101 (2007).

${ }^{16}$ F. Oulton, V. J. Sorger, T. Zentgraf, R. Ma, C. Gladden, L. Dai, G. Bartal, and X. Zhang, Nature 461(7264), 629 (2009).

${ }^{17}$ C. Wu, C. Kuo, C. Wang, C. He, M. Lin, H. Ahn, and S. Gwo, Nano Lett. 11(10), 4256 (2011).

${ }^{18}$ Y. J. Lu, J. Kim, H. Y. Chen, C. Wu, N. Dabidian, C. E. Sanders, C. Y. Wang, M. Y. Lu, B. H. Li, and X. Qiu, Science 337(6093), 450 (2012).

${ }^{19}$ Lumerical, see www.lumerical.com.

${ }^{20}$ T. Yang, S. Goto, M. Kawata, K. Uchida, A. Niwa, and J. Gotoh, Jpn. J. Appl. Phys., Part 2 37, L1105 (1998).

${ }^{21}$ E. D. Palik, Handbook of Optical Constants of Solids (Academic, 1998). 